\title{
Fire acupuncture for mild to moderate knee osteoarthritis: a protocol for a randomized controlled pilot trial
}

\author{
Yuan-Bo Fu' ${ }^{1,2}$, Bin Li ${ }^{1}$, San-Feng Sun ${ }^{2}$, Hui-Lin Liu', Xin Wang ${ }^{1}$, Shao-Song Wang ${ }^{1}$, Fan Zhang ${ }^{1}$, Xin Du', \\ Du-Juan Ge Gei Shang $^{2}$, Rui-Li Liang ${ }^{2}$, Li-Na Wang ${ }^{2}$, Fang Yuan', Jing-Qing Sun ${ }^{1 *+}$ and Jun-Wei Chen ${ }^{1,3^{*+}}$ (D)
}

\begin{abstract}
Background: Knee osteoarthritis $(\mathrm{KOA})$ is one of the most common bone and joint diseases. As one of the main non-drug therapies, acupuncture is widely used to treat KOA, although the evidence for its efficacy is inconclusive. The objective of this pilot trial is to clarify the clinical efficacy and safety of fire acupuncture in the treatment of mild to moderate KOA and to provide high-quality data for further research.

Methods/design: This study is a prospective randomized controlled pilot trial in which 120 patients with mild to moderate KOA will be randomly allocated in equal proportions to a fire acupuncture group or a general acupuncture group. They will receive acupuncture for six sessions over 2 weeks. The primary end point is success rate, which will be calculated based on the change from baseline of the pain and function scores in the Western Ontario and McMaster Universities Osteoarthritis Index at 4 weeks. Secondary end points include the proportion of patients achieving clinical improvement based on: (1) the OMERACT-OARSI responder criteria, (2) levels of matrix metalloproteinase 3, interleukin $1 \beta$, and tumor necrosis factor a in blood, and (3) a subjective efficacy evaluation from patients.
\end{abstract}

Trial registration: Chinese Clinical Trial Registry, ChiCTR1800019162. Registered on 29 October 2018.

Keywords: Knee Osteoarthritis, Fire Acupuncture, Basic health management

\section{Background}

Knee osteoarthritis (KOA) is one of the most common musculoskeletal disorders [1]. Its prevalence rate is $49 \%$ among people over 60 and $80 \%$ among people over 75 in China. One third of people over 65 suffer from KOA, according to epidemiological surveys [2]. Worldwide, an estimated 25,000 people suffer from KOA [3]. Most have radiological or clinical evidence of osteoarthritis [4], and the incidence of the disease has been increasing with the aging of the population [5]. Its high incidence and high disability rate not only bring a heavy economic burden to patients, but also seriously affect their quality of life [6]. If symptoms persist, patients may have low levels of

\footnotetext{
* Correspondence: sjqdoctor@163.com; 847431304@qq.com

${ }^{+}$Jing-Qing Sun and Jun-Wei Chen contributed equally to this work.

${ }^{1} T$ The Department of Acupuncture and Moxibustion, Beijing Hospital of

Traditional Chinese Medicine, Capital Medical University, Beijing Key

Laboratory of Acupuncture Neuromodulation, No. 23 Meishuguanhou Street,

Dongcheng District, Beijing 100010, China

Full list of author information is available at the end of the article
}

social activity and abnormal psychological functioning, which may eventually lead to a decline in the quality of life and even an inability to live independently, causing heavy psychological pressure and a burden on the patient and their family.

Analgesics-such as non-steroidal anti-inflammatory drugs, opioids, and non-opioid central analgesics (such as tramadol)-are still recommended to treat the disease, according to the guidelines for the diagnosis and treatment of KOA published by the American Academy of Orthopaedic Surgeons in 2013 [7] and those published by the National Institute for Health and Care Excellence in 2014 [8]. However, drug treatments can cause serious gastrointestinal complications, such as ulcers, perforations, and bleeding, and the risk increases with age and treatment duration. At present, the treatment for $\mathrm{KOA}$ is pain relief, symptomatic treatment, maintenance, or improvement of joint function, since there is no effective way to completely block or reverse the development of 
KOA. Most patients with advanced KOA have to "cure" it by undergoing knee replacement. Unfortunately, many KOA patients are unable to undergo a knee replacement due to their physical condition or affordability. Therefore, a safe and effective therapy for KOA that is free of side effects is urgently required to improve patients' quality of life and reduce the national medical burden.

Acupuncture is important in traditional Chinese medicine as a non-drug therapy. It has been used for more than 2000 years in China [9]. In fire acupuncture (FA), a heated needle is inserted into an acupuncture point to eliminate the symptoms of a disease [10]. A metaanalysis published in Evidence-Based Complementary and Alternative Medicine showed that FA has a higher response rate and higher cure rate in the treatment of KOA [11].

Although FA is widely used to treat KOA and has achieved good results, there is still a lack of good medical evidence to verify whether it is beneficial. In addition, the molecular biological mechanism is still unclear. Fire needle therapy has been developed over thousands of years. It has a wide range of clinical applications in China, with a definite curative effect and a wide range of indications. Studies have shown that FA can reduce the level of some inflammatory cytokines that are elevated in KOA, such as interleukin 1 (IL-1), interleukin 6 (IL-6), and tumor necrosis factor $\alpha$ (TNFa) [12].

Nevertheless, none of the 13 studies included in the meta-analysis in Evidence-Based Complementary and Alternative Medicine [11] were of sufficient quality. Most of the research into FA quoted in the literature is of poor quality, for example, the randomization methodology is often unclear and there can be issues with the selection of the control group. Thus, doctors and patients may have concerns about the efficacy and safety of FA. Therefore, high-quality prospective randomized controlled trials are needed to determine the clinical efficacy and safety of FA in treating mild and moderate KOA and to explore the mechanisms of action.

\section{Methods/design}

\section{Study design}

The study is a prospective multi-center parallel randomized controlled trial. We will enroll patients from Beijing Hospital of Traditional Chinese Medicine (Capital Medical University) and Beijing Huairou District Hospital of Traditional Chinese Medicine with mild to moderate $\mathrm{KOA}$, according to the inclusion and exclusion criteria, from January 2019 to December 2019. Participants will be randomly allocated to one of two groups and will receive treatment for 2 weeks. The trial has been registered with the Chinese Clinical Trial Registry (ChiCTR1800019162). All items from the World Health Organization's trial registration data set have been entered into the registry. The intervention includes six sessions of acupuncture and two follow-up visits (Fig. 1 and Table 1).

\section{Inclusion criteria}

To be eligible, patients must meet all of the following inclusion criteria:

Aged 38-85 years (either sex)

Meet the diagnostic criteria for primary KOA

Mild to moderate KOA (Kellgren-Lawrence score 1-2)

Able to receive FA treatment

Voluntary participation in the study

Able and willing to sign the informed consent form

\section{Exclusion criteria}

To be eligible, patients must not meet any of the following inclusion criteria:

1. Secondary KOA

2. The knee joint has a history of trauma or surgery in the past year

3. Severe joint deformity (varus or valgus $\geq 8^{\circ}$ )

4. Injection of glucocorticoid into the articular cavity of the target knee within 3 months

5. Injection of sodium hyaluronate into the articular cavity of the target knee within 2 weeks

6. Have received acupuncture or moxibustion for symptomatic hip arthritis or patellar arthritis on the same side of the target knee joint within 2 weeks

7. Symptomatic hip or patellar arthritis on the same side of the target knee joint

8. Inflammatory or other rheumatic disease

9. Acute knee meniscus injury

10. Acute knee injury or fracture around the knee

11. Tumor around the knee

12. Tuberculosis or idiopathic osteonecrosis

13. Taking paracetamol, non-steroidal antiinflammatory drugs, or analgesics for other diseases

14. Uncontrolled hypertension or diabetes

15. Severe cardiovascular, pulmonary, liver, spleen, kidney, or hematopoietic disease

16. Tumor hemorrhages

17. Mental illness

18. Scar diathesis or skin sensory impairment

19. Pregnant or lactating women

\section{Randomization and allocation concealment}

Random permuted blocks will be used. The allocation to the FA group and to the general acupuncture (GA) group will be in a 1:1 ratio. Opaque numbered envelopes will be used to conceal the allocation. Researchers will open the envelopes according to the order of enrollment. The acupuncturists will not be involved in the randomization. Data collectors, data 


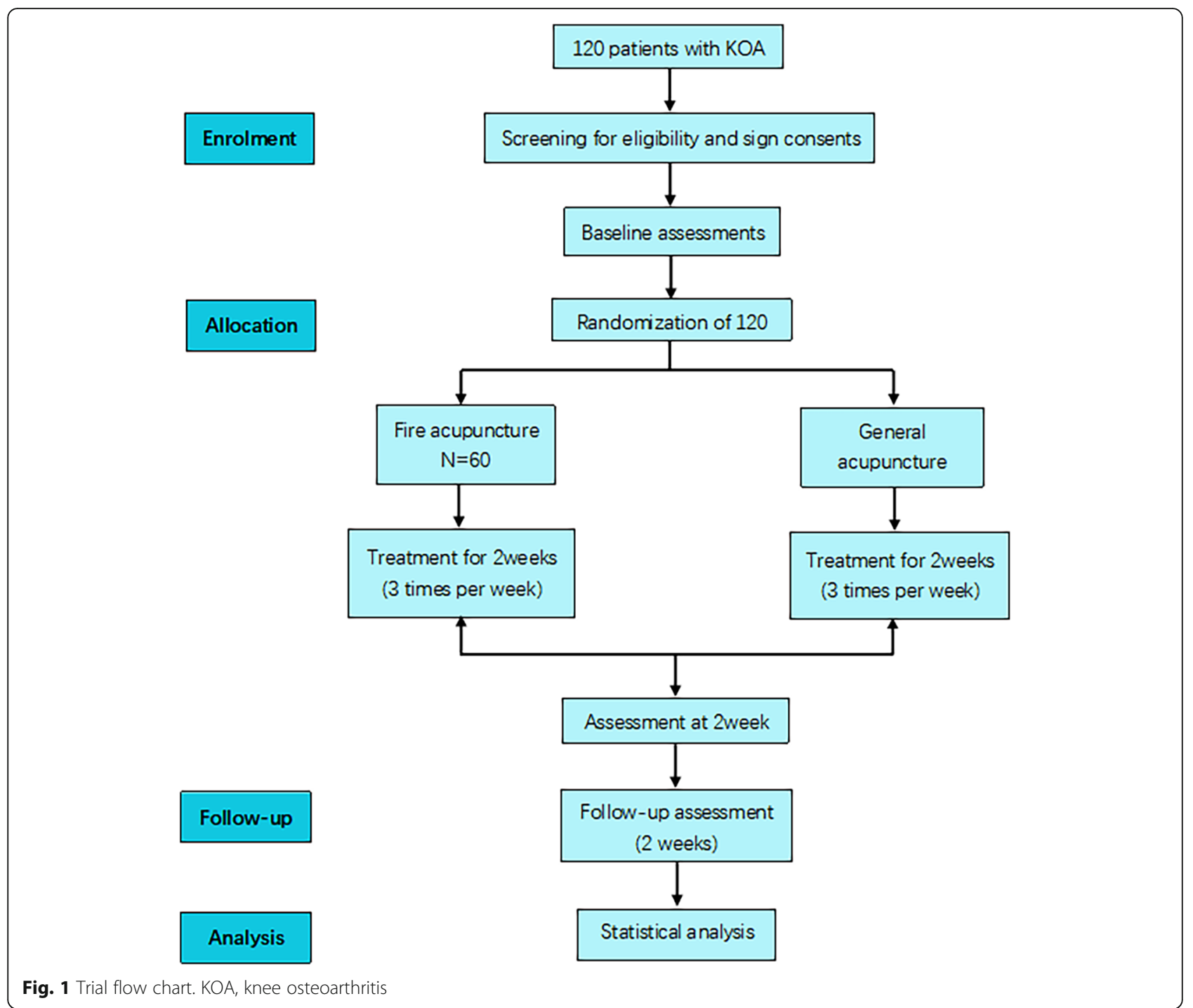

evaluators, and statisticians will be blinded to group assignment. Participants will not be blinded.

\section{Interventions}

This study conforms to the CONSORT [13] and STRICTA [14] criteria. All acupuncturists are required to have had Chinese medical practitioner licenses for at least 20 years and they will receive the same training under the clinical treatment program prior to the study.

The acupuncture sessions will be for $30 \mathrm{~min}$. There will be three sessions per week for 2 weeks, giving six sessions in total. Sterilized medium and coarse He's fire needles will be used for the FA group. Disposable, sterile steel, $0.30 \times 50 \mathrm{~mm}$ acupuncture needles (Ande disposable acupuncture needles, Guizhou Medical Co., Guizhou, China) will be used for the GA group. If participants experience pain of intensity $\geq 4$ on a $10 \mathrm{~cm}$ visual analog scale [15], they will be given Celebrex.
Adverse reactions during treatment will be recorded in the case report form.

Patients in both groups will receive basic health management. Blood tests will be performed on day 0 and the second week after the six acupuncture sessions. In this study, we strengthened patients' self-management by giving them a detailed manual on managing KOA. The manual was developed by the research team based on the second edition of the evidence-based medical guidelines for KOA published by the American Academy of Orthopaedic Surgeons. The manual explains the natural course of the disease, gives advice on how to avoid psychological effects, and describes the drugs used and their side effects. The manual recommends that they (1) maintain good posture, (2) avoid being stationary for a long time, (3) do not run or jump, and (4) minimize their use of stairs, etc. The contents of the manual are explained to the patient in detail before randomization. 
Table 1 Schedule of enrollment, interventions, and assessments

\begin{tabular}{|c|c|c|c|c|}
\hline & \multirow{2}{*}{$\begin{array}{l}\text { Baseline } \\
-1 \text { day }\end{array}$} & \multirow{2}{*}{$\begin{array}{l}\text { Treatment phase } \\
0 \text { day }\end{array}$} & \multicolumn{2}{|c|}{ Follow-up phase } \\
\hline & & & 2 weeks & 4 weeks \\
\hline \multicolumn{5}{|l|}{ Patients } \\
\hline Informed consent & $\times$ & & & \\
\hline Sign informed consent & & $x$ & & \\
\hline Medical history & $\times$ & & & \\
\hline Physical examination & $x$ & & & \\
\hline Randomization & & $x$ & & \\
\hline $\begin{array}{l}\text { Intervention } \\
\text { FA group }(n=60)\end{array}$ & Six sessions of FA & & & \\
\hline $\begin{array}{l}\text { Comparisons } \\
\text { GA group }(n=60)\end{array}$ & Six sessions of GA & & & \\
\hline \multicolumn{5}{|l|}{ Outcomes } \\
\hline WOMAC & & $x$ & $x$ & $x$ \\
\hline OMERACT-OARSI & & $x$ & $x$ & $x$ \\
\hline MMP-3 & & $\times$ & $x$ & \\
\hline$I L-1 \beta$ & & $\times$ & $\times$ & \\
\hline TNF-a & & $\times$ & $x$ & \\
\hline SEE & & $\times$ & $\times$ & $x$ \\
\hline \multicolumn{5}{|l|}{ Participant safety } \\
\hline Adverse events & & $\times$ & $x$ & $x$ \\
\hline
\end{tabular}

FA fire acupuncture, GA general acupuncture, IL interleukin, MMP-3 matrix metalloproteinase 3, SEE subjective efficacy evaluation, TNF- $a$ tumor necrosis factor a, WOMAC Western Ontario and McMaster Universities Osteoarthritis Index

Subjects were asked to comply with these selfmanagement recommendations for at least 4 weeks after being randomized. Patients with severe pain may take non-steroidal anti-inflammatory drugs, including topical drugs and systemic drugs. They are asked to record the drug used, the frequency of administration, the dosage, and any adverse drug reactions in the manual.

\section{FA group}

Acupuncture points are selected based on the $b i$ syndrome in traditional Chinese medicine. There are five local points (ST34, ST35, SP10, EX-LE4, and GB34) and one or two pain points (ashi points) (Fig. 2). The procedure is as follows:

1. Place the patient in a supine position with their knees bent.

2. Disinfect the acupuncture points.

3. Heat the tip and middle of the acupuncture needle until it glows red

4. Pierce each point three times to a depth of about $0.5 \mathrm{~cm}$, moving the needle quickly in and out. The needle will be heated before each insertion.
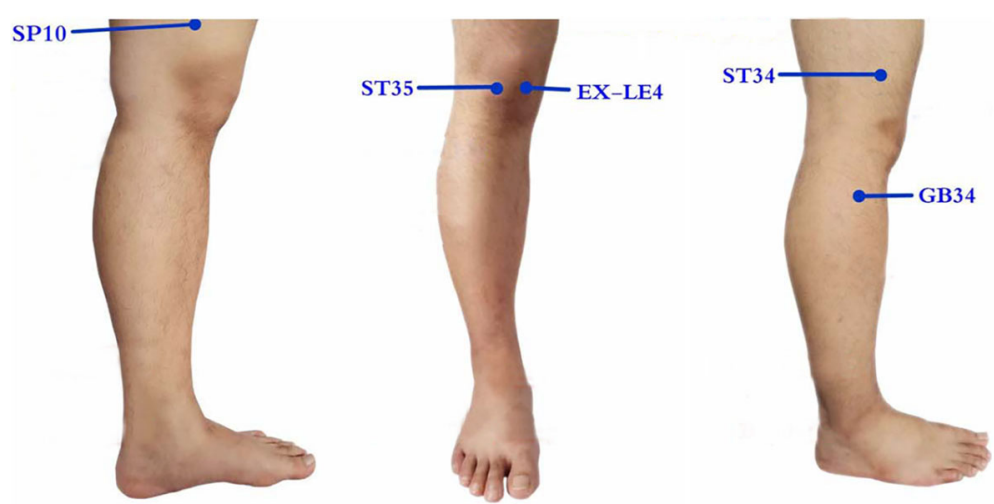

Fig. 2 Acupuncture points for both groups 
5. Press the acupuncture eyes for a moment with a sterile dry cotton ball.

\section{GA group}

2The acupuncture points used are the same as those for the FA group. The procedure is as follows:

1. Place the patient in a supine position with their knees bent.

2. Disinfect the acupuncture points.

3. Insert a needle into each point to the optimum depth. The needles remain in place for approximately $30 \mathrm{~min}$.

4. Remove all needles and use sterile cotton balls to avoid bleeding.

Acupuncturists are instructed to achieve de qi and to stimulate the needles manually for at least $10 \mathrm{~s}$.

\section{Primary outcome}

The success rate will be calculated based on the change from the baseline in the total score for the Western Ontario and McMaster Universities Osteoarthritis Index (WOMAC) $[16,17]$ at weeks 2 and 4 , which has five dimensions: (1) pain, (2) joint stiffness, (3) joint physiology, (4) social functioning, and (5) emotion, with a total score of 24. Higher scores represent a worse condition.

\section{Secondary outcomes}

1. The proportion of patients with a clinical improvement: The OMERACT-OARSI responder criteria will be used at weeks 2 and 4 to assess whether a patient has a clinical improvement, and compare whether there is a difference in improvement between the two groups (Fig. 3).

2. Subjective efficacy evaluation: At weeks 2 and 4, a research assistant will ask each patient about the effects of the therapy they received on their KOA compared to the baseline. The evaluation is scored as follows: greatly aggravated -3 , moderately aggravated -2 , mildly aggravated -1 , totally unhelpful 0 , very little help 1 , moderate assistance 2 , and great help 3 .

3. Laboratory indicators: Patients' peripheral blood will be collected at weeks 0 and 2 , and levels of matrix metalloproteinase 3 (MMP-3), IL-1 $\beta$, and TNF- $\alpha$ will be measured by an enzyme-linked immunosorbent assay.

\section{Sample size}

Since no previous multicenter randomized controlled trials have studied the efficacy of FA in treating KOA, this study will provide effect size data for sample size calculations in subsequent large-scale randomized controlled trials. Overall, 120 outpatients and inpatients will be recruited at the two research centers, giving 60 participants per group.

\section{Statistical analysis}

This project will use a remote data capture system. Data will be double entered by two clerks. Once locked, the SAS database will be delivered to the statisticians along with a description of the data structure and a coding table. The statistical analysis will be performed by statisticians who are blinded to the allocation and are not involved in other aspects of the study.

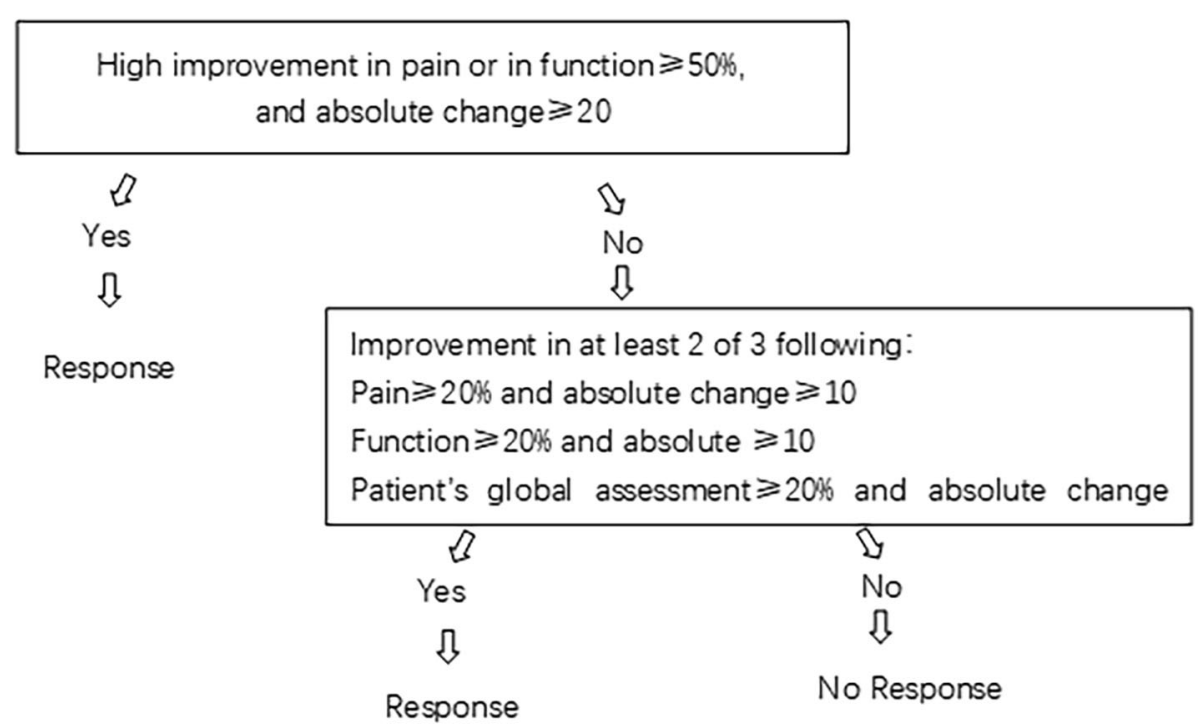

Fig. 3 OMERACT-OARSI responder criteria 
All randomized subjects will be analyzed statistically according to the intention-to-treat principle. The last observation carried forward method will be used to impute missing values. The software SPSS (V.18.0 KO for Windows) will be used to analyze the results. All statistical tests will be two sided, and $p<0.05$ will be considered statistically significant. Continuous data will be described by mean \pm standard deviation, median, maximum, and minimum. Discrete data will be described by frequencies and percentages. Continuous data will be analyzed by a paired $t$-test (if normally distributed) or a non-parametric test (if not normally distributed). Discrete data will be analyzed with a Pearson chi-squared test or a rank sum test.

The cartilage exfoliation rate due to adverse events will be determined for each center and for each group. The exfoliation rates will be analyzed with Fisher's exact test. Using the case report forms, we will evaluate whether each participant received the prescribed dose and number of treatments according to the research plan.

An effectiveness analysis will be conducted using the randomized populations. Participants who have not received any treatment and participants who have received treatment but for whom there is no valid evaluation data will be considered as missing and will be included in the effectiveness analysis.Participants who cannot tolerate treatment after the start of observation, have serious side effects, have less than 2 weeks of treatment, or are lost to follow-up will be considered as cases of shedding. When the participant falls off, the investigator should make an appointment for follow-up, telephone or letter to contact the participant as much as possible, ask the reason, and record the last treatment time to complete the evaluation project. For trial cases withdrawn due to adverse reactions and ineffective treatment, the investigator should take corresponding treatment measures according to the actual situation of the subject. The investigator should indicate the main reason for terminating the trial. If the exfoliation rate exceeds $20 \%$ of the total number of participants, the study will be considered a failure and terminated.

\section{Quality supervision and management}

To improve patient compliance with the treatment and health management plans, we propose to use the patient manuals, regular mobile phone reminders and other methods, and patients will be compensated financially. The data monitoring committee (DMC) consists of clinical experts, statisticians, experimenters, third-party inspectors, etc. The DMC will analyze summarized data by the third-party blind method to ensure the authenticity and reliability of the research results. The DMC will monitor the quality of the study. It is independent of the sponsors and there are no competing interests. Specialized institutions designated by the funders will conduct a test acceptance and funding audit.
The clinical trial will be suspended or discontinued if the safety of any participant is compromised or if any serious adverse reactions are not reported within the prescribed time. If the trial is suspended or discontinued, the participants, the project funder (Beijing Huairou District Science and Technology Committee), and the ethics committee will be informed of the reason.

\section{Patient and public involvement}

We will make details of the study available to the public through online publicity and posters. Interested patients can visit our research center before participating in the study to learn more about it. Patients will not be involved in the design of the study or recruitment.

\section{Ethics and dissemination \\ Patient consent and dissemination policy}

This protocol has been registered with the Chinese Clinical Trial Registry. All participants will be given ample time to consider whether to participate in the study and to sign the informed consent, which includes consent for blood tests. All blood samples taken from participants will be destroyed after the trial. Participants can withdraw their informed consent at any time during the study. We will publish the results of the trial in a peerreviewed clinical journal for wide dissemination. Participants who experience severe adverse reactions or irreversible injuries as a result of the trial will be reasonably compensated, according to the circumstances.

\section{Access to data and confidentiality}

Only research team members have access to the research data. All data will be kept strictly confidential during the study. On completion of the study, participants will receive a personal summary of the data collected in the study.

\section{Discussion}

$\mathrm{KOA}$ is a common disease with a high disability rate. It results in significant financial losses and is a burden for patients and society. At present, the treatment of $\mathrm{KOA}$ is still at the level of pain relief and symptomatic treatment, and the etiology and pathogenesis of KOA are still unclear [18]. Studies have shown that the main pathological changes of KOA are the degeneration of the articular cartilage and a change in synovial composition [19].

As a major non-drug therapy, acupuncture is widely used to treat KOA. Studies have shown that acupuncture can effectively relieve pain and improve the mobility of the knee [11]. FA is a combination of acupuncture and heat. It has a good curative effect on musculoskeletal diseases by warming channels to clear collaterals and veins. Studies have shown that FA can reduce the level of some inflammatory cytokines that are elevated in 
KOA [12]. Therefore, we suspect that KOA may be related to changes in some inflammatory factors.

Compared with other clinical treatments, FA has the advantages of significant efficacy and high safety, and moreover, it is readily accepted for the treatment of KOA by patients. Although a published meta-analysis [10] showed that FA has advantages in KOA treatment, high-quality randomized controlled trials investigating FA as well as research based on disease classification or with stratification are still lacking. Therefore, this prospective randomized parallel controlled trial is necessary to assess the safety and efficacy of FA in treating KOA and to indicate the mechanisms of action.

A carefully designed clinical trial has a suitable control group. We know from the literature review as well as our own clinical experience that GA is also effective for KOA. It is also a relatively safe non-pharmacological treatment that is available in most countries. Thus, we used GA for the control group rather than another treatment that would not be as effective. In this study, the participants in both groups will receive three acupuncture sessions a week for 2 weeks, for a total of six sessions.

The target disease of this study is mild and moderate KOA. Therefore, if this study proves that FA is effective in treating KOA, the next step is to evaluate the efficacy of FA in treating severe KOA. In addition, this study is an initial exploration of the correlation between FA and inflammatory factors in peripheral blood. If this correlation is verified, then the mechanism for how FA affects KOA can be further explored from the perspective of molecular biology (Additional file 1).

\section{Strengths}

In principle, FA has been recognized in China as a relatively safe non-drug treatment. This study will provide further high-quality clinical evidence on the safety of FA. The planned study has the potential to demonstrate that FA is a novel, practical, and effective treatment for KOA. If $\mathrm{FA}$ is effective in alleviating pain and improving joint function in patients with KOA, then it could be adopted as a cheap non-drug therapy instead of potentially addictive drugs or surgery, which may help in reducing the living and economic burden on patients. Since FA is simple, fast, cheap, and easy to implement, then if its efficacy and safety in treating KOA are recognized, doctors will have more treatment options for KOA.

\section{Limitations}

Acupuncturists and participants will not be blinded due to the nature of the intervention. Participants can not be blinded to the study design due to the feeling of the skin during treatment. The acupuncturists will not be involved in assessing outcomes or the data analysis.

Although the duration of treatment for each participant is only 4 weeks, it is possible to compare the efficacy and short-term effect of FA and GA. Due to the short observation time, however, it will not be possible to determine the long-term efficacy of using fire needles. Levels of the inflammatory factors in peripheral blood may increase during the follow-up period rather than decrease as we expect.

\section{Trial status}

This trial is currently recruiting participants (protocol version 2.0, 20 April 2019). The study will run from 1 June 2019 to 31 March 2020.

\section{Supplementary information}

Supplementary information accompanies this paper at https://doi.org/10. 1186/s13063-019-3744-2.

Additional file 1. SPIRIT 2013 checklist.

\begin{abstract}
Abbreviations
DMC: Data monitoring committee; FA: Fire acupuncture; GA: General acupuncture; IL: Interleukin; KOA: Knee osteoarthritis; MMP-3: Matrix metalloproteinase 3; SEE: Subjective efficacy evaluation; TNF-a: Tumor necrosis factor a; WOMAC: Western Ontario and McMaster Universities Osteoarthritis Index
\end{abstract}

\section{Authors' contributions}

YF and JC conceived and designed the study. YF received funding for this trial. SS, LH, XW, and SW polished and revised the manuscript. FZ, XD, LS, and DG provided suggestions on ethics. RL, LW, JS, BL, and FY provided methodological recommendations. JC and YF drafted the manuscript. All authors contributed to the refinement of the study protocol and approved the final manuscript.

\section{Funding}

This work was supported by the Beijing Huairou District Science and Technology Plan Project, Beijing Key Laboratory of Acupuncture Neuromodulation, Beijing, China (BZ0437, award NY2019-6) and the Beijing Municipal Hospital Research and Development Program (award PZ2019019).

\section{Availability of data and materials}

The original data will be released within 6 months of completion of the trial. The specific disclosure method for the original data will be selected according to the research process.

Ethics approval and consent to participate

Ethical approval of this study has been granted by the research ethics committee of Beijing Hospital of Traditional Chinese Medicine, Capital Medical University (permission number 2018SB-076). Written informed consent will be obtained from all participants.

Central ethical approval has been given by the research ethics committee of Beijing Hospital of Traditional Chinese Medicine, Capital Medical University (approval 2018SB-076) and we will not begin recruiting to the trial at other centers until local ethical approval has been obtained.

Consent for publication

Not applicable.

Competing interests

The authors declare that they have no competing interests. 


\section{Author details}

The Department of Acupuncture and Moxibustion, Beijing Hospital of Traditional Chinese Medicine, Capital Medical University, Beijing Key Laboratory of Acupuncture Neuromodulation, No. 23 Meishuguanhou Street, Dongcheng District, Beijing 100010, China. ${ }^{2}$ Beijing Huairou District Hospital of Traditional Chinese Medicine, No. 1 Houheng Street, Huairou District, Beijing 101400, China. ${ }^{3}$ Beijing University of Chinese Medicine, No. 11, Bei San Huan Dong Lu, Chaoyang District, Beijing 100029, China.

Received: 22 July 2019 Accepted: 23 September 2019

Published online: 04 December 2019

\section{References}

1. Hochberg MC, Altman RD, April KT, et al. American College of Rheumatology 2012 recommendations for the use of nonpharmacologic and pharmacologic therapies in osteoarthritis of the hand, hip, and knee. Arthritis Care Res (Hoboken). 2012;64(4):465-74.

2. Mavrommatis $\mathrm{Cl}$, Argyra E, Vadalouka A, et al. Acupuncture as an adjunctive therapy to pharmacological treatment in patients with chronic pain due to osteoarthritis of the knee: a 3-armed, randomized, placebo-controlled trial. Pain. 2012;153(8):1720-6. https://doi.org/10.1016/j.pain.2012.05.005.

3. O'Neill TW, McCabe PS, McBeth J. Update on the epidemiology, risk factors and disease outcomes of osteoarthritis. Best Pract Res Clin Rheumatol. 2018; 32(2):312-26. https://doi.org/10.1016/j.berh.2018.10.007 [published Online First: 2018/12/12].

4. Goldring MB, Goldring SR. Osteoarthritis. J Cell Physiol. 2007;213(3):626-34 https://doi.org/10.1002/jcp.21258.

5. Baker K, McAlindon T. Exercise for knee osteoarthritis. Curr Opin Rheumatol. 2000;12(5):456-63.

6. Higgs R. Osteoarthritis: Concentrated efforts to detect early OA. Nat Rev Rheumatol. 2010;6(11):616. https://doi.org/10.1038/nrrheum.2010.165.

7. Brown GA. AAOS clinical practice guideline: treatment of osteoarthritis of the knee: evidence-based guideline, 2nd edition. J Am Acad Orthop Surg. 2013;21(9):577-9. https://doi.org/10.5435/JAAOS-21-09-577.

8. National Clinical Guideline Centre. Osteoarthritis: Care and Management in Adults. London; 2014. https://www.ncbi.n/m.nih.gov/pubmed/25340227.

9. Wu JN. A short history of acupuncture. J Altern Complement Med. 1996;2(1): 19-21. https://doi.org/10.1089/act.1996.2.19.

10. China National Standardization Administration SAoQS, Inspection and Quarantine. Standardized manipulations of acupuncture and moxibustionPart 12:Fire acupuncture. Beijing: Standards Press of China; 2009.

11. Wang $Y$, Xie $X$, Zhu $X$, et al. Fire-needle moxibustion for the treatment of knee osteoarthritis: a meta-analysis. Evidence-Based Complement Alternat Med. 2016;2016:1392627. https://doi.org/10.1155/2016/1392627.

12. Hu QS, Zhang QR, Jia CS, et al. Clinical randomized controlled observation of the treatment of senile knee osteoarthritis with needle acupuncture and its effect on inflammatory cytokines in knee joint cavity. Acupuncture Research. 2011;(02):110-5.

13. Schulz KF, Altman DG, Moher D, et al. CONSORT 2010 statement: updated guidelines for reporting parallel group randomised trials. BMJ. 2010;340: c332. https://doi.org/10.1136/bmj.c332.

14. MacPherson H, Altman DG, Hammerschlag R, et al. Revised Standards for Reporting Interventions in Clinical Trials of Acupuncture (STRICTA): extending the CONSORT statement. PLoS Med. 2010;7(6):e1000261. https:// doi.org/10.1371/journal.pmed.1000261.

15. Liu CZ, Xie JP, Wang LP, et al. A randomized controlled trial of single point acupuncture in primary dysmenorrhea. Pain Med. 2014;15(6):910-20. https:// doi.org/10.1111/pme.12392.

16. Bellamy N, Buchanan WW, Goldsmith $\mathrm{CH}$, et al. Validation study of WOMAC: a health status instrument for measuring clinically important patient relevant outcomes to antirheumatic drug therapy in patients with osteoarthritis of the hip or knee. J Rheumatol. 1988;15(12):1833-40.

17. Goldsmith $\mathrm{CH}$, Boers $\mathrm{M}$, Bombardier C, et al. Criteria for clinically important changes in outcomes: development, scoring and evaluation of rheumatoid arthritis patient and trial profiles. OMERACT Committee. J Rheumatol. 1993; 20(3):561-5

18. Gobbi A, Lad D, Karnatzikos G. The effects of repeated intra-articular PRP injections on clinical outcomes of early osteoarthritis of the knee. Knee Surg Sports Traumatol Arthrosc. 2015;23(8):2170-7. https://doi.org/10.1007/ s00167-014-2987-4.
19. Aigner T, Kim HA. Apoptosis and cellular vitality: issues in osteoarthritic cartilage degeneration. Arthritis Rheum. 2002;46(8):1986-96. https://doi.org/ 10.1002/art.10554

\section{Publisher's Note}

Springer Nature remains neutral with regard to jurisdictional claims in published maps and institutional affiliations.
Ready to submit your research? Choose BMC and benefit from:

- fast, convenient online submission

- thorough peer review by experienced researchers in your field

- rapid publication on acceptance

- support for research data, including large and complex data types

- gold Open Access which fosters wider collaboration and increased citations

- maximum visibility for your research: over $100 \mathrm{M}$ website views per year

At $\mathrm{BMC}$, research is always in progress.

Learn more biomedcentral.com/submissions 